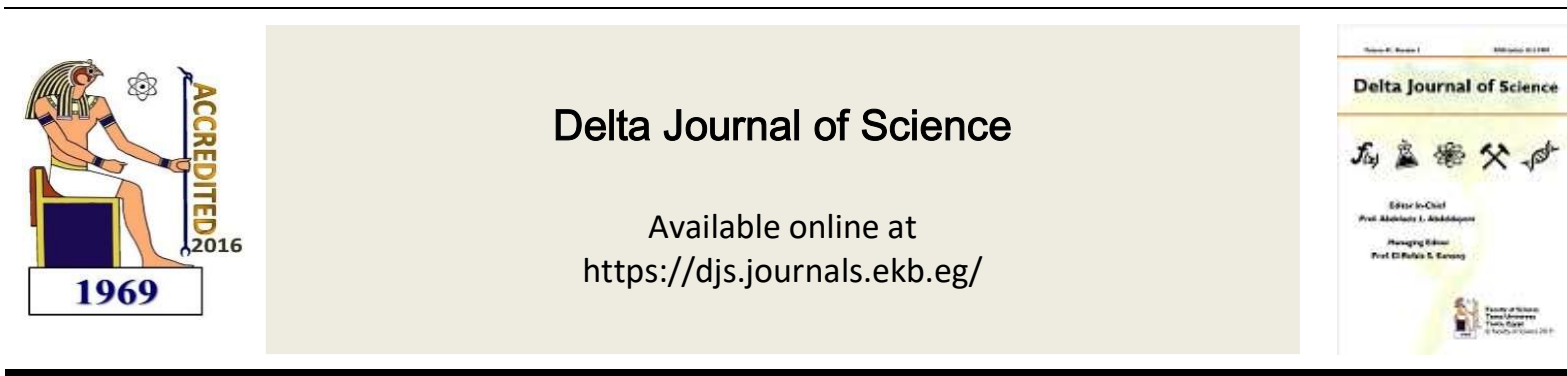

Research Article

MATHEMATICS

\title{
The Solution of the Fractional Form of Unsteady Axisymmetric Squeezing Fluid Flow with Slip and No-Slip Boundaries by Analytical Techniques
}

\author{
A. A. Hemeda and I. A. Lairje* \\ Department of Mathematics, Faculty of Science, Tanta University, Tanta 31527, Egypt \\ * Correspondence: A. A. Hemeda; Department of Mathematics, Faculty of Science, Tanta University, \\ Tanta 31527,Egypt; Email: aahemeda@yahoo.com
}

\section{KEY WORDS}

Squeezing flow; Axisymmetric flow; Variational iteration method;

Adomian

decomposition method; New iterative method; Picard method and Fractional Calculus

\begin{abstract}
In this article, two powerful techniques called variational iteration and Adomian decomposition methods are proposed to solve analytically the fractional form of the fourth order nonlinear ordinary differential equation which reduced by similarity transformation from the basic system of nonlinear partial differential equations of motion of an unsteady axisymmetric flow of nonconducting, Newtonian fluid squeezed between two circular plates with slip and no-slip boundaries. The analysis of convergence of the proposed methods is discussed through the absolute residual errors for various Reynolds number and various values of the fractional order. Comparisons between the results obtained by the proposed methods with those obtained by the new iterative and Picard methods are made which confirm that the proposed methods are powerful methods and therefore suitable for solving this kind of problems.
\end{abstract}

\section{Introduction}

The importance of fluid flow through channels began since long time ago in various applications in the life specially in agriculture. Recently, squeezing flow has large attention of the researchers and scientists due to its wide range of applications in various fields like chemical, food industries and in biomechanics Practical applications of squeezing flows in the mentioned fields are modeling of lubrication systems, compression and injection modeling and polymer processing. These flows are induced by applying vertical velocities or normal stresses by means of a moving boundary, that can be frequently noted in many hydro-dynamical tools and machines.

The first work in squeezing flows was laid down by Stefan [1] in which he developed an ad-hoc asymptotic solution of Newtonian fluids. An explicit solution of the squeeze flow, considering inertial terms, has done by Thorp [2]. However, Gupta and Gupta showed that the solution in [2] fails to satisfy 
boundary conditions [3]. In [4] Ran et. al. used the homotopy analysis method to obtain an explicit series solution for the squeezing flow between two infinite plates. Also, by this method in [5] Rashidi et. al. established an analytical solution to the unsteady squeezing flow of a second-grade fluid between two circular plates. Moreover, the processes of polymer extrusion are modeled by squeezing flow of viscous fluids in [6]. Leider and Bird in [7] presented theoretical analysis of powerlaw fluid between parallel disks. Verma [8] and Singh et. al. [9] have established numerical solutions of squeezing flow between parallel plates. Hemeda and Aladdad in [10] used the new iterative and Picard methods for solving the fractional form of unsteady axisymmetric flow of nonconducting, Newtonian fluid squeezed between two circular plates with slip and noslip boundaries. Qayyum et. al. present, in [11] analysis of unsteady axisymmetric flow of nonconducting, Newtonian fluid squeezed between two circular plates with slip and noslip boundaries using OHAM, while in [12], the authors modeling and analyzing the unsteady axisymmetric flow of nonconducting, Newtonian fluid squeezed between two circular platers passing through porous medium channel with slip boundary condition using HPM. Sheikholeslami et. al. in [13] used the Adomian decomposition method to solve the problem governing the unsteady flow of a nanofluid squeezed between two parallel plates and in [14] used the HPM for solving the problem governing the heat transfer of a nanofluid flow squeezed between parallel plates. For more studies, you can see [15-18].

The differential equations of fractional order, are the generalized type of the classical differential equations of integer order. Recently, the fractional differential equations have been the focus of many researchers because of their frequent appearance in many applications in viscoelasticity, physics, biology, engineering and fluid mechanics. Therefore, considerable attention has been given to the solutions of differential equations of fractional order, of fluid mechanics and physics interest. Most nonlinear differential equations of fractional order do not have exact analytic solutions, so numerical and approximation techniques, such as Jacobi collocation method [19], Galerkin method [20], He's frequency-amplitude formulation and energy balance methods [21], max-min and Hamiltonian methods [22, 23], variational iteration method [24-28] and Adomian decomposition method [29-33] must be used. The variational iteration and Adomian decomposition methods, which are special techniques of the homotopy analysis method [34], are relatively new techniques to provide analytical approximation to nonlinear problems and they are particularly valuable as tools for researchers, because they provide immediate and visible symbolic terms of analytic solutions, as well as numerical approximate solutions to nonlinear differential equations without linearization or discretization.

In last years, the application of the proposed methods in extended for fractional differential equations. The goal of this chapter, is to prepare and use these methods for solving the fractional order form of an unsteady axisymmetric flow of nonconducting, Newtonian fluid squeezed between two circular plates with slip and no-slip boundaries because of the importance of this problem and its wide applications in many subjects in fluid mechanic. In addition, the comparisons between the results obtained by the proposed methods with those obtained by some other methods are considered to confirm the power and efficiency of these methods in solving this kind of problems.

\section{Formulation of the Problem}

In this section, the unsteady axisymmetric flow of incompressible Newtonian fluid with viscosity $\mu$, density $\rho$ and kinematic viscosity $v$, squeezed between two circular plates having speed $E_{w}(t)$ is considered with a fractional form. At any time $t$, it is assumed that the distance between the two circular plates is $2 h(t)$. Also, it is assumed that $r$-axis is the central axis of the channel while $z$-axis is taken normal to it. Plates move symmetrically with respect to the central axis 
$z=0$ while the flow is axisymmetric about $r=0$. The normal and longitudinal velocity components in axial and radial directions are $w_{z}(r, z, t)$ and $w_{r}(r, z, t)$, respectively (Figure $\left.E_{14}\right) .($ For more details, see [10-12].

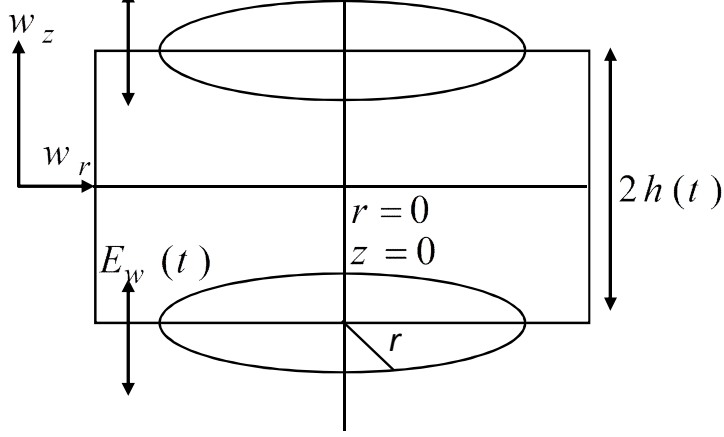

Fig. 1: Geometrical of the problem.

The equations of motion are:

$$
\begin{aligned}
& \frac{\partial w_{r}}{\partial r}+\frac{w_{r}}{r}+\frac{\partial w_{z}}{\partial z}=0, \\
& \frac{\partial p}{\partial r}+\rho\left(\frac{\partial w_{r}}{\partial t}-w_{z} \Omega\right)=-\mu \frac{\partial \Omega}{\partial z}, \\
& \frac{\partial p}{\partial z}+\rho\left(\frac{\partial w_{r}}{\partial t}+w_{r} \Omega\right)=\frac{\mu}{r} \frac{\partial}{\partial r}(r \Omega),
\end{aligned}
$$

where $\Omega=\Omega(r, z, t)$ is the vorticity function; and $p=p(r, z, t)$ is the generalized pressure.

The boundary conditions on $w_{r}(r, z, t)$ and $w_{z}(r, z, t)$ are:

$w_{r}(r, z, t)=0, \quad w_{z}(r, z, t)=E_{w}(t)$ at $z=h$

$\frac{\partial}{\partial z} w_{r}(r, z, t)=0, w_{z}(r, z, t)=0$ at $z=0$,

where $E_{w}(t)=\frac{d h}{d t}$ is the velocity of the plates. The boundary conditions in equation (4) are because of no-slip at the upper plate when $z=h$ and symmetry at $z=0$. If we define the dimensionaless parameter:

$$
\eta=\frac{z}{h(t)}
$$

equations (1), (2) and (3) transforms to:

$$
\begin{aligned}
& \frac{\partial w_{r}}{\partial r}+\frac{w_{r}}{r}+\frac{1}{h} \frac{\partial w_{z}}{\partial \eta}=0, \\
& \frac{\partial p}{\partial r}+\rho\left(\frac{\partial w_{r}}{\partial t}-w_{z} \Omega\right)=-\frac{\mu}{h} \frac{\partial \Omega}{\partial \eta} \\
& \frac{1}{h} \frac{\partial p}{\partial \eta}+\rho\left(\frac{\partial w_{r}}{\partial t}+w_{r} \Omega\right)=\frac{\mu}{r} \frac{\partial}{\partial r}(r \Omega) .
\end{aligned}
$$

The boundary conditions on $w_{r}$ and $w_{z}$ are:

$$
\begin{array}{lrr}
w_{r}=0, & w_{z}=E_{w}(t) & \text { at } \eta=1 \\
\frac{\partial}{\partial \eta} w_{r}=0, & w_{z}=0 & \text { at } \eta=0 .
\end{array}
$$

By eliminating he generalized pressure between equations (7) and (8), we obtain:

$$
\rho\left[\frac{\partial \Omega}{\partial t}+w_{r} \frac{\partial \Omega}{\partial r}+\frac{w_{z}}{h} \frac{\partial \Omega}{\partial \eta}-\frac{w_{r}}{r} \Omega\right]=\mu\left[\nabla^{2} \Omega-\frac{\Omega}{r^{2}}\right],
$$

where $\nabla^{2}$ is the Laplacian operator.

Defining velocity components as $[3,5]$ :

$w_{r}=-\frac{r}{2 h(t)} E_{w}(t) u^{\prime}(\eta), \quad w_{z}=E_{w}(t) u(\eta)$,

we see that equation (6) is identically satisfied and equation (10) becomes:

$\frac{d^{4} u}{d \eta^{4}}+R\left[(\eta-u) \frac{d^{3} u}{d \eta^{3}}+2 \frac{d^{2} u}{d \eta^{2}}\right]-Q \frac{d^{2} u}{d \eta^{2}}=0$,

where

$R=\frac{h E_{w}}{v}$ and $Q=\frac{h^{2}}{v E_{w}} \frac{d E_{w}}{d t}$.

In equations (12) and (13) $R$ and $Q$ are functions of $t$ but for similarity solution we consider these functions are constants. Since $E_{w}=\frac{d h}{d t}$. Integrate first equation of equation (13), we get:

$$
h(t)=(c t+d)^{\frac{1}{2}} \text {, }
$$


where $c$ and $d$ are constants. The plates move from each other symmetrically with respect to $\eta$ when $c>0$ and $d>0$. Also, the plates approach to each other and squeezing flow exists with similar velocity profiles when $c<0, d>0$ and $h(t)>0$. From equations (13) and (14) it follows that $Q=-R$. Therefore equation (12) becomes:

$\frac{d^{4} u}{d \eta^{4}}+R\left[(\eta-u) \frac{d^{3} u}{d \eta^{3}}+3 \frac{d^{2} u}{d \eta^{2}}\right]=0$.

Using equations (9) and (11), we can define the boundary conditions at the upper plate in case of no-slip and slip as follows:

No-slip at the wall:

$u(1)=1, \quad u^{\prime}(1)=0, \quad u(0)=0, \quad u^{\prime \prime}(0)=0$

Slip at the wall:

$u(1)=1, \quad u^{\prime}(1)=u^{\prime \prime}(1), u^{\prime}(0)=0, \quad u^{\prime \prime}(0)=0$.

\section{Fractional Calculus}

Now we mention basic definitions of fractional calculus which are used in the present work.

Definition 3.1: The fractional integral operator of order $\alpha>0$, of a function $f(t) \in c_{\mu}$ and $\mu \geq 1$, in the RiemannLiouville sense is defined as [35]:

$$
\begin{aligned}
& I_{t}^{\alpha} f(t)=\frac{1}{\Gamma(\alpha)} \int_{0}^{t}(t-r)^{\alpha-1} f(\tau) d \tau, \quad \alpha>0, t>0 \\
& I_{t}^{0} f(t)=f(t) .
\end{aligned}
$$

For the Riemann-Liouville fractional integral operator, $I_{t}^{\alpha}$, we obtain:

$I_{t}^{\alpha} t^{v}=\frac{\gamma(v+1) t^{v+\alpha}}{\Gamma(v+1+\alpha)}$.

Definition 3.2: The fractional derivative of $f(t)$ in the Caputo sense is defined as [36]:

$$
\begin{aligned}
& D_{t}^{\alpha} f(t)=I^{m-\alpha} D^{t m} \\
& =\frac{1}{\Gamma(m-\alpha)} \int_{0}^{t}(t-r)^{m-\alpha-1} f^{(m)}(\tau) d \tau
\end{aligned}
$$

For the Caputo fractional derivative operator, $D_{t}^{\alpha}$, we obtain:

$D_{t}^{\alpha} t^{v}=\frac{\Gamma(v+1) t^{v-\alpha}}{\Gamma(v+1-\alpha)}$.

For the Riemann-Liouvill fractional integral and Caputo fractional derivative of order $\alpha$, we have the following relations:

Lemma 1: If $m-1<\alpha \leq m, m \in N$ and $f \in C_{\mu}^{m}, \mu \geq-1$, then:

$$
\begin{aligned}
& D_{t}^{\alpha} I_{t}^{\alpha} f(t)=f(t), \\
& D_{t}^{\alpha} I_{t}^{\alpha} f(t)=\left\{\begin{array}{lr}
f(t)-\sum_{k=0}^{m-1} f^{(k)}\left(0_{+}\right) \frac{t^{k}}{k !}, t>0, & m-1<\operatorname{deg} \cdot f(t) \\
0, & m-1 \geq \operatorname{deg} \cdot f(t)
\end{array}\right.
\end{aligned}
$$

Remark 1: According to the previous fractional calculus, equation (15) can be rewrite in the following fractional order form:

$$
\frac{d^{\alpha} u}{d \eta^{\alpha}}+R\left[(\eta-u) \frac{d^{3} u}{d \eta^{3}}+3 \frac{d^{2} u}{d \eta^{2}}\right]=0,3<\alpha \leq 4 .
$$

\section{Analysis of the Considered Methods}

In this section, we discuss the considered methods with preparing them for solving any fractional differential equation.

\subsection{Variational Iteration Methods (VIM):}

To illustrate the basic idea of this method, let us consider the following general differential equation of fractional order [24-28]:

$D_{t}^{\alpha} u(t)=R[t] u(t)+g(t), \quad m-1<\alpha \leq m, m \in N$

subject to the initial values:

$$
\frac{d^{k}}{d t^{k}} u(0)=h_{k}, \quad k=0,1,2, \ldots, m-1,
$$

where $R[t]$ is a nonlinear differential operator in $t, g(t), h_{k}(t)$ are given continuous functions and $D_{t}^{\alpha}$ is the fractional differential operator of order $\alpha>0$ in the 
Caputo sense. According to the VIM, we can construct the correction functional for equation (23) as:

$$
\begin{aligned}
& u_{n+1}(t)=u_{n}(t)+I_{t}^{\beta}\left[\lambda\left[D_{t}^{\alpha} u_{n}(t)-R[t] \tilde{u}_{n}(t)-g(t)\right)\right], \\
& =u_{n}(t)+\frac{1}{\Gamma(\beta)} \int_{0}^{t}(t-\tau)^{\beta-1} \lambda(\tau)\left[D_{t}^{\alpha} u_{n}(\tau)-R[\tau] \tilde{u}_{n}(\tau)\right. \\
& -g(\tau))] d \tau,
\end{aligned}
$$

where $I_{t}^{\beta}$ is the fractional integral operator o Riemann-Liovill of order $\beta=\alpha+1-m$, which respect to $t$ and $\lambda$ is a general Lagrange multiplier, which can be identified optimally via variational theory, to identify $\lambda$ approximately, we must made some approximation. Therefore, the correction functional (24) can be approximately in the form:

$u_{n+1}(t)=u_{n}(t)+\int_{0}^{t}\left[\lambda(\tau)\left(D_{t}^{\alpha} u_{n}(\tau)-R[\tau] \tilde{u}_{n}(\tau)-g(\tau)\right)\right] d \tau$

Here we apply restricted variations to the nonlinear term $R[t] u$, in this case we can easily determine the multiplier. Making the above functional stationary, noticing that $\delta \tilde{u}_{n}=0$,

$\delta u_{n+1}(t)=\delta u_{n}(t)+\delta \int_{0}^{t} \lambda(\tau)\left[D_{t}^{\alpha} u_{n}(\tau)-g(\tau)\right] d \tau$,

yield the following Lagrange multiplier:

$$
\left\{\begin{array}{lll}
\lambda(\tau)=-1, & \text { for } & m=1, \\
\lambda(\tau)=\tau-t, & \text { for } & m=2, \\
\lambda(\tau)=-\frac{1}{2}(\tau-t)^{2}, & \text { for } & m=3, \\
\lambda(\tau)=\frac{1}{6}(\tau-t)^{3}, & \text { for } & m=4 .
\end{array}\right.
$$

Generally, and in closed form, equation (27) becomes:

$\lambda(\tau)=\frac{(-1)^{m}}{\Gamma(m)}(\tau-t)^{m-1}, \quad m \geq 1$.

Therefore, for $m=1(0<\alpha \leq 1)$, we substitute $\lambda=-1$ into (24) to obtain the iterative formula:

$u_{n+1}(t)=u_{n}(t)-I_{t}^{\alpha}\left[D_{t}^{\alpha} u_{n}(t)-R[\tau] u_{n}(t)-g(t)\right], n \geq 0$
For $m=2(1<\alpha \leq 2)$, we substitute $\lambda=\tau-t$ into (24) to obtain the iterative formula:

$u_{n+1}(t)=u_{n}(t)-(\alpha-1) I_{t}^{\alpha}\left[D_{t}^{\alpha} u_{n}(t)-R[\tau] u_{n}(t)-g(t)\right], n \geq 0$

For $m=3(2<\alpha \leq 3)$, we substitute $\lambda=-\frac{1}{2}(\tau-t)^{2}$ into (24) to obtain the iterative formula:

$u_{n+1}(t)=u_{n}(t)-\frac{1}{2}\left(\alpha^{2}-3 \alpha+2\right) I_{t}^{\alpha}\left[D_{t}^{\alpha} u_{n}(t)-R[\tau] u_{n}(t)-g(t)\right], n \geq 0$

Finally, for $m=4(3<\alpha \leq 4)$, we substitute $\lambda=\frac{1}{6}(\tau-t)^{3}$ into (24) to obtain the iterative formula:

$$
\begin{gathered}
u_{n+1}(t)=u_{n}(t)-\frac{1}{6}\left(\alpha^{3}-6 \alpha^{2}+11 \alpha-6\right) I_{t}^{\alpha}\left[D_{t}^{\alpha} u_{n}(t)-R[\tau] u_{n}(t)\right. \\
-g(t)], n \geq 0 .
\end{gathered}
$$

The zero solution $u_{0}$ can be freely chosen if it satisfies most of the given conditions. However, the success of the method depends on the proper selection of the zero solution $u_{0}$. Finally, we approximate the solution $u(t)=\lim _{n \rightarrow \infty} u_{n}(t)$ by the $n^{\text {th }}$-term $u_{n}(t)$.

\subsection{Adomian Decomposition Method (ADM):}

To illustrate the basic idea of this method, let us consider the fractional differential equation [29-33]:

$D_{t}^{\alpha} u(t)=L u(t)+N u(t)+g(t), \quad m-1<\alpha \leq m, m \in N$

with the initial values:

$\frac{d^{k}}{d t} u(0)=h_{k}, \quad k=0,1,2, \ldots, m-1$,

where $L$ is linear operator and $N$ is nonlinear operator. The method is based on applying the fractional integral operator $I_{t}^{\alpha}$, the inverse of the fractional differential operator $D_{t}^{\alpha}$, to both sides of (33) to obtain:

$u(t)=\sum_{k=0}^{m-1} h_{k} \cdot \frac{t^{k}}{k !}+I_{t}^{\alpha}[L u(t)+N u(t)+g(t)]$ 
The ADM suggests the solution $u(t)$ be decomposed into the infinite series of components:

$u(t)=\sum_{n=0}^{\infty} u_{n}(t)$

and the nonlinear term $N u(t)$ in equation (33a) is decomposed as:

$N u=\sum_{n=0}^{\infty} A_{n}$,

where $A_{n}$ are the so-called Adomian polynomials. Substituting the decomposition series (35) and (36) into both side of (34) gives:

$\sum_{n=0}^{\infty} u_{n}(t)=\sum_{k=0}^{m-1} h_{k} \cdot \frac{t^{k}}{k !}+I_{t}^{\alpha}\left[L \sum_{n=0}^{\infty} u_{n}(t)+\sum_{n=0}^{\infty} A_{n}+g(t)\right]$.

According to the ADM, we can introduce the recurrence relation

as:

$u_{0}(t)=\sum_{k=0}^{m-1} h_{k} \cdot \frac{t^{k}}{k !}+I_{t}^{\alpha}[g(t)]$,

$u_{j+1}(t)=I_{t}^{\alpha}\left[L u_{j}(t)+A_{j}\right], \quad j \geq 0$,

The polynomial $A_{n}$ of Adomian can be calculated for all nonlinear terms via specific constructed by Adomian. Therefore, the general form of $A_{n}$ is:

$$
A_{n}=\frac{1}{n !}\left[\frac{d^{n}}{d \lambda^{n}} N\left(\sum_{k=0}^{n} \lambda^{k} u_{k}\right)\right]_{\lambda=0} .
$$

This formula is easy to compute. Finally, we approximate the solution $u(t)$ by the truncated series:

$$
\begin{aligned}
& \phi_{N}(t)=\sum_{j=0}^{N-1} u_{j}(t) \quad \text { and } \\
& \lim _{N \rightarrow \infty} \phi_{N}(t)=u(t) .
\end{aligned}
$$

\section{Applications:}

In the following, we illustrate the application of the two-considered method to solve the nonlinear fractional order ordinary differential equation (22) subject to the boundary conditions (16).

\subsection{VIM:}

Using Equations (22) and (16), the initial value fractional order problem:

$\frac{d^{\alpha}}{d t^{\alpha}} u(\eta)+R\left[(\eta-n) \frac{d^{3} u}{d \eta^{3}}+3 \frac{d^{2} u}{d \eta^{2}}\right]=0,3<\alpha \leq 4$,
$u(0)=0, \quad u^{\prime}(0)=a, \quad u^{\prime \prime}(0)=0, \quad u^{\prime \prime \prime}(0)=b$,

according to the iteration formula (32), becomes:

$u_{n+1}(h)=u_{n}(\eta)-\frac{1}{6}\left(\alpha^{3}-6 \alpha^{2}+11 \alpha-6\right) I_{\eta}^{\alpha}$

$\left.R\left(\left(\eta-u_{n}\right) \frac{d^{3} u_{n}}{d \eta^{3}}+3 \frac{d^{2} u_{n}}{d \eta^{2}}\right)\right]$.

Beginning with the initial solution $u_{0}(\eta)=a \eta+\frac{b \eta^{3}}{6}$, which satisfies the given initial conditions, we can obtain the following first few components of the solution:

$$
\begin{aligned}
& u_{1}(\eta)=a \eta+\frac{b \eta^{3}}{6}-\frac{1}{6}\left(\alpha^{3}-6 \alpha^{2}+11 \alpha-6\right)\left(\frac{4 b R^{2} \eta^{1+\alpha}}{\Gamma(2+\alpha)}-\frac{a b R \eta^{1+\alpha}}{\Gamma(2+\alpha)}\right. \\
& \left.-\frac{b^{2} R \eta^{3+\alpha}}{\Gamma(4+\alpha)}\right), \\
& u_{2}(\eta)=u_{1}+\frac{1}{6^{2}}\left(\alpha^{3}-6 \alpha^{2}+11 \alpha-6\right)^{2}\left(\frac{4 b R^{2}(\alpha-1) \eta^{-1+2 \alpha}}{\Gamma(2 \alpha)}\right. \\
& -\frac{5 a b R^{2}(\alpha-1) \eta^{-1+2 \alpha}}{\Gamma(2 \alpha)}-\frac{2 b^{2} R^{2} \Gamma(2+\alpha) \eta^{1+2 \alpha}}{3 \Gamma(-1+\alpha) \Gamma(2+2 \alpha)} \\
& \left.\frac{a^{2} b^{2} R^{2} \Gamma(2+\alpha) \eta^{1+2 \alpha}}{6 \Gamma(-1+\alpha) \Gamma(2+2 \alpha)}+\ldots\right), \\
& u_{3}(\eta)=a \eta+\frac{b \eta^{3}}{6}-\frac{1}{6}\left(\alpha^{3}-6 \alpha^{2}+11 \alpha-6\right)\left(\frac{4 b R \eta^{1+\alpha}}{\Gamma(2+\alpha)}-\frac{a b R \eta^{1+\alpha}}{\Gamma(2+\alpha)}\right. \\
& \left.-\frac{b^{2} R \eta^{3+\alpha}}{\Gamma(4+\alpha)}\right)+\frac{1}{6^{2}}\left(\alpha^{3}-6 \alpha^{2}+11 \alpha-6\right)^{2}\left(\frac{4 b R^{2}(\alpha-1) \eta^{-1+2 \alpha}}{\Gamma(2 \alpha)}\right. \\
& \left.\frac{5 a b R^{2}(\alpha-1) \eta^{-1+2 \alpha}}{\Gamma(2 \alpha)}+\ldots\right),
\end{aligned}
$$

and so on. In the same way, we can obtain the higher components of the solution. 
I the special case, $\alpha=4$, equation (43) gives:

$$
\begin{aligned}
& u(\eta)=a \eta+\frac{b \eta^{3}}{6}-\frac{b R \eta^{5}}{30}+\frac{a b R \eta^{5}}{120}+\frac{b R \eta^{7}}{5040}+\frac{b R^{2} \eta^{7}}{210}-\frac{a b R^{2} \eta^{7}}{280} \\
& +\frac{a^{2} b R^{2} \eta^{7}}{1680}-\ldots
\end{aligned}
$$

Using the boundary conditions in equation (16) with the initial conditions in equation (41), the unknown's $a$ and $b$ for fixed values of $R$ in equation (44) can be easily determined. In case of no-slip boundary, then $a=1.5$ and $b=-30$. For $R=0.5$, the solution is:

$$
\begin{aligned}
& u(\eta)=1.5 \eta-0.5 \eta^{3}+0.03125 \eta^{5}+0.000334821 \eta^{7}-0.000171673 \eta^{9} \\
& +1.02166 \times 10^{-6} \eta^{11}+1.36077 \times 10^{-6} \eta^{13}-2.6881 \times 10^{-8} \eta^{15} \\
& -2.68729 \times 10^{-9} \eta^{17}+1.23186 \times 10^{-10} \eta^{19}+3.42474 \times 10^{-12} \eta^{21} \\
& -1.79838 \times 10^{-13} \eta^{23}-4.69921 \times 10^{-15} \eta^{25}+7.12005 \times 10^{-17} \eta^{27} \\
& +1018728 \times 10^{-18} \eta^{29}+1.18008 \times 10^{-20} \eta^{31},
\end{aligned}
$$

and in case of slip boundary with $\gamma=1$, then $a=0.75$ and $b=1.5$. For $R=0.5$, the solution is:

$u(\eta)=0.75 \eta-0.25 \eta^{3}+0.0203125 \eta^{5}+0.00113002 \eta^{7}-8.87674 \times 10^{-5} \eta^{9}$ $+6.753994 \times 10^{-6} \eta^{11}-4.50029 \times 10^{-7} \eta^{13}+2.54254 \times 10^{-8} \eta^{15}$ $-1.00801 \times 10^{-9} \eta^{17}+3.48556 \times 10^{-11} \eta^{19}-1 . .05106 \times 10^{-12} \eta^{21}$ $+2.53779 \times 10^{-14} \eta^{23}-4.22141 \times 10^{-16} \eta^{25}+4.23058 \times 10^{-18} \eta^{27}$ $-2.22146 \times 10^{-20} \eta^{29}+4.6097 \times 10^{-23} \eta^{31}$

\subsection{ADM:}

According to the recurrence relation (38), the initial value fractional order problem (41) gives:

$$
\begin{aligned}
& u_{0}(\eta)=a \eta+\frac{b \eta^{3}}{6}, \\
& u_{j+1}(\eta)=-I_{t}^{\alpha}\left[R\left(t u_{j}^{\prime \prime \prime}+3 u_{j}^{\prime \prime}-A_{j}\right)\right], \quad j \geq 0,
\end{aligned}
$$

and therefore, the first few components of the solution are as follows:

$$
\begin{aligned}
& u_{0}(\eta)=a \eta+\frac{b \eta^{3}}{6} \\
& u_{1}(\eta)=\frac{4 b R \eta^{\alpha+1}}{\Gamma(2+\alpha)}+\frac{a b R \eta^{\alpha+1}}{\Gamma(2+\alpha)}+\frac{b^{2} R \eta^{3+\alpha}}{\Gamma(4+\alpha)}, \\
& u_{2}(\eta)=\frac{4 b R^{2}(\alpha-1) \eta^{-1+2 \alpha}}{\Gamma(2 \alpha)}-\frac{a b R^{2}(\alpha-1) \eta^{-1+2 \alpha}}{\Gamma(2 \alpha)}-\frac{b^{2} R^{2}(1+\alpha) \eta^{1+2 \alpha}}{\Gamma(2+2 \alpha)} \\
& +\frac{12 b R^{2} \eta^{-1+2 \alpha}}{\Gamma(2 \alpha)}-\frac{3 a b R^{2} \eta^{-1+2 \alpha}}{\Gamma(2 \alpha)}-\ldots
\end{aligned}
$$

etc. In the same way, we can be obtain the higher components of the solution. The 4term approximate solution is

$$
\begin{aligned}
& u(\eta)=\sum_{i=0}^{3} u_{i}(\eta) \\
& =a \eta+\frac{b \eta^{3}}{6}+\frac{a b R \eta^{1+\alpha}}{\Gamma(2+\alpha)}-\frac{4 b R \eta^{1+\alpha}}{\Gamma(2+\alpha)}+\frac{b^{2} R^{2} \eta^{3+\alpha}}{\Gamma(2+\alpha)} \\
& +\frac{4 b R^{2}(\alpha-1) \eta^{-1+2 \alpha}}{\Gamma(2 \alpha)}-\frac{a b R^{2}(\alpha-1) \eta^{-1+2 \alpha}}{\Gamma(2 \alpha)} \\
& -\frac{b^{2} R^{2}(\alpha+1) \eta^{1+2 \alpha}}{\Gamma(2 \alpha)}+\ldots
\end{aligned}
$$

In the special case, $\alpha=4$, equation (48) gives:

$$
\begin{aligned}
& u(\eta)=a \eta+\frac{b \eta^{3}}{6}-\frac{b R \eta^{5}}{30}+\frac{a b R \eta^{5}}{120}+\frac{b^{2} R \eta^{7}}{5040}+\frac{b R^{2} \eta^{7}}{210}-\frac{a b R^{2} \eta^{7}}{280} \\
& +\frac{a^{2} b R^{2} \eta^{7}}{1680}-\ldots
\end{aligned}
$$

Similarly, using the boundary conditions in equation (16) with the initial conditions in equation (41), the solution for no-slip boundary, at $R=0.5$, is:

$$
\begin{aligned}
& u(\eta)=1.5 \eta-0.5 \eta^{3}+0.03125 \eta^{5}+0.000334821 \eta^{7}+0.000171673 \eta^{9} \\
& +1.02166 \times 10^{-6} \eta^{11}+1.48018 \times 10^{-6} \eta^{13} \\
& +4.88258 \times 10^{-8} \eta^{15}
\end{aligned}
$$

and for slip boundary, at $R=0.5$, is

$$
\begin{aligned}
& u(\eta)=0.75 \eta+0.25 \eta^{3}-0.0203125 \eta^{5}+0.00113002 \eta^{7}-8.87674 \times \\
& 10^{-5} \eta^{9}+6.75394 \times 10^{-6} \eta^{11}-2.78821 \times 10^{-7} \eta^{13} \\
& +3.05161 \times 10^{-9} \eta^{15} .
\end{aligned}
$$


It is clear that the number of terms of the solution obtained by ADM in equations (48), (49), (50) and (51) are less than the number obtained by VIM in equations (43), (44), (45) and (46).

The residual error of the problem is:

$$
\operatorname{Re}(\eta)=\text { Residual Error }=\frac{d^{\alpha} \hat{u}}{d \eta^{\alpha}}+R\left[(\eta-\hat{u}) \frac{d^{3} \hat{u}}{d \eta^{3}}+3 \frac{d^{2} \hat{u}}{d \eta^{2}}\right],
$$

where $\hat{u}$ is the 4-term approximate solution in equation (43) or equation (48) for equation (41).

If $\operatorname{Re}(\eta)=0$, then the approximate solution $\hat{u}$ becomes the exact solution $u$. However, in nonlinear problems, this does not occur.

It is clear from the obtained results that the above considered methods are used simply and accurately without linearization or discretisation with their difficulties. Therefore, these methods are powerful methods for solving the nonlinear fractional order differential equations.

\subsection{NIM and PM:}

The initial value fractional order problem (41) have been solved by Hemeda and Eladdad in [10] using the new iterative method (NIM); introduced by Daftaedar-Gejji and Jafari and Picard method (PM); introduced by Emile Picard, where the two methods are powerful techniques to give analytical approximate solutions for nonlinear problems and they are particularly valuable as tools for researchers, because they give immediate and visible symbolic terms of analytic solutions, as well as numerical approximate solutions to nonlinear differential equations without lineariza-tion or discretisation. The 4-term approximate solutions at $\alpha=4.0, R=0.5$, in case of no-slip boundary with $a=1.5, b=-3.0$, takes the form:

$$
\begin{aligned}
& u(\eta)=1.5 \eta-0.5 \eta^{3}+0.03125 \eta^{5}+0.000334821 \eta^{7}-0.000171673 \eta^{9} \\
& +1.02166 \times 10^{-6} \eta^{11}+1.36077 \times 10^{-6} \eta^{13}-2.6881 \times 10^{-8} \eta^{15} \\
& -2.68729 \times 10^{-9} \eta^{17}+1.23186 \times 10^{-10} \eta^{19}+3.42474 \times 10^{-12} \eta^{21} \\
& -1.79838 \times 10^{-13} \eta^{23}-4.69921 \times 10^{-15} \eta^{25}+7.12005 \times 10^{-17} \eta^{27}
\end{aligned}
$$

$$
+2.18728 \times 10^{-18} \eta^{29}+1.18008 \times 10^{-20} \eta^{31},
$$

and in case of slip boundary with $\gamma=1, a=0.75, b=1.5$, takes the form:

$$
\begin{aligned}
& u(\eta)=0.75 \eta+0.25 \eta^{3}-0.023125 \times 10^{-6} \eta^{115}+0.00113002 \times 10^{-6} \eta^{117} \\
& -8.87674 \times 10^{-5} \eta^{9}+6.75394 \times 10^{-6} \eta^{11}-4.50029 \times 10^{-7} \eta^{13} \\
& +2.4254 \times 10^{-8} \eta^{15}-1.00801 \times 10^{-9} \eta^{17}+3.48554 \times 10^{-11} \eta^{19} \\
& -1.05106 \times 10^{-12} \eta^{21}+2.53779 \times 10^{-14} \eta^{23}-4.22141 \times 10^{-16} \eta^{25} \\
& +4.23058 \times 10^{-18} \eta^{27}-2.22146 \times 10^{-23} \eta^{29}+4.6097 \times 10^{-23} \eta^{31}
\end{aligned}
$$

It is clear that the solutions obtained by VIM in equations (45) and (46) are the same solutions as obtained y NIM and PM in equations (53) and (54) respectively which means that the three methods are identical in solving this problem while, the solution obtained by ADM in equations (50) and (51) is with simple formulation than these methods. Also, it is clear that the solutions obtained by VIM and NIM and PM are polynomials of degree 31, while the solution obtained by $\mathrm{ADM}$ is a polynomial of degree 15 which means that the ADM is simpler in its procedures and computations than these methods.

\section{Numerical Results and Discussion:}

In this chapter, the unsteady axisymmetric flow of nonconducting, incompressible Newtonian fluid squeezed between two circular plates is considered. The resulting nonlinear fractional order boundary value problem are solved analytically in cases of no-slip and slip boundaries using the VIM and ADM.

Tables 1, 2, and 3 represent the approximate solutions in case of no-slip boundary for different values of the fractional order $\alpha$ at fixed value of the Reynolds number $R$ with the corresponding absolute residual errors $|\operatorname{Re}|$ at $\alpha=4.0$ in each table, while Tables 4, 5 , and 6 are in case of slip boundary at the slip parameter $\gamma=1$. It is clear from the results in the size tables that as $\alpha \rightarrow 4, u_{\mathrm{VIM}} \rightarrow u_{\mathrm{ADM}}$. Therefore, the approximate solutions converge to the exact solutions for this problem. Also, the six tables 
indicate that the $|\operatorname{Re}|$ for VIM, in case of noslip boundary, are less than that for ADM, while the inverse in case of slip boundary.

Moreover, Tables 7, 8, and 9, reflect the comparisons between the $|\operatorname{Re}|$ of the VIM,

methods are identical in solving this problem, while the $\gamma=1.0$ are less for ADM in case of slip boundary than those for the other three methods and more in case of no-slip boundary.

Figure 2a shows the residual errors, Re, for various Reynolds number $R=0.2,0.4,0.6$ at $\alpha=0.4$, in case of no-slip boundary, obtained by VIM, while Figure $2 b$, in case of slip boundary, obtained by ADM with $\gamma=0.9$. In addition to the above Figures, the effect of Reynolds number $R$ on velocity profiles by VIM in case of no-slip boundary is depicted in Figures 3. In these profiles, $R$ is varied as $R=0.2,0.4,0.6$ and noted that the normal velocity is increased with increasing $R$ (Figure 3a). Also, it is observed that the
ADM with those for the NIM and PM [9] for various values of $R=0.1,0.3,0.5$, in case of no-slip and slip boundaries at $\alpha=4.0$, $\gamma=1.0$. It is clear from the results in the three tables that the $\gamma=1.0$ are equal for VIM, NIM and PM which means that the three normal velocity monotonically increases from $\eta=0$ to $\eta=1$ for fixed $R$ at $a$ given time. Figure $3 \mathrm{~b}$ illustrates the effect of $R$ on the longitudinal velocity. It is clear that this component of velocity decreases near the wall but increases near the central axis of the channel.

The effect of Reynolds number $R$ on velocity profiles by ADM in case of slip boundary is shown in Figures 4 for $R=0.2,0.4,0.6$ at fixed slip parameter $\gamma=0.9$. It is observed that the normal velocity decreases as $R$ increases (Figure 4a). Also, it is noted that the longitudinal velocity decreases near the central axis of the channel but increases near the walls when $R$ increases (Figure 4b).

Table 1: Solution for different values of $\eta$ and $\alpha$ at $R=0.2$, and the corresponding absolute residual errors at $\alpha=4.0$ in case of no-slip boundary by the two methods.

\begin{tabular}{ccccccc}
\hline \multirow{2}{*}{$\eta$} & \multicolumn{3}{c}{ VIM } & \multicolumn{3}{c}{ ADM } \\
\cline { 2 - 7 } & $\alpha=\mathbf{3 . 9}$ & $\alpha=\mathbf{4 . 0}$ & $|\boldsymbol{R e}|$ & $|\boldsymbol{R e}|$ & $\alpha=\mathbf{4 . 0}$ & $\alpha=\mathbf{3 . 9}$ \\
\hline 0.0 & 0.000000 & 0.000000 & 0.000000 & 0.000000 & 0.0000000 & 0.0000000 \\
0.1 & 0.149500 & 0.149500 & $9.00113^{-14}$ & $3.69149^{-14}$ & 0.149500 & 0.149500 \\
0.2 & 0.296005 & 0.296004 & $1.15501^{-11}$ & $1.66173^{-11}$ & 0.296004 & 0.296006 \\
0.3 & 0.436531 & 0.436530 & $1.83062^{-10}$ & $9.63263^{-10}$ & 0.436530 & 0.436541 \\
0.4 & 0.568138 & 0.568128 & $9.57782^{-10}$ & $1.55055^{-8}$ & 0.568128 & 0.0568167 \\
0.5 & 0.687912 & 0.687893 & $4.23159^{-10}$ & $1.34707^{-7}$ & 0.687893 & 0.687998 \\
0.6 & 0.793009 & 0.792979 & $3.88958^{-8}$ & $8.06399^{-7}$ & 0.792979 & 0.793220 \\
0.7 & 0.880653 & 0.880622 & $3.19774^{-7}$ & $3.75394^{-6}$ & 0.880622 & 0.881103 \\
0.8 & 0.948155 & 0.948148 & $1.71883^{-6}$ & $1.45616^{-5}$ & 0.948148 & 0.949021 \\
0.9 & 0.992923 & 0.992998 & $7.29223^{-6}$ & $4.91485^{-5}$ & 0.992998 & 0.994466 \\
1.0 & 1.012480 & 1.012740 & $2.62701^{-5}$ & $1.48578^{-4}$ & 1.012740 & 1.015070 \\
\hline
\end{tabular}

Table 2: Solution for different values of $\eta$ and $\alpha$ at $R=0.4$, and the corresponding absolute residual errors at $\alpha=4.0$ in case of no-slip boundary by the two methods.

\begin{tabular}{ccccccc}
\hline \multirow{2}{*}{$\eta$} & \multicolumn{3}{c}{ VIM } & \multicolumn{3}{c}{ ADM } \\
\cline { 2 - 7 } & $\alpha=\mathbf{3 . 9}$ & $\alpha=\mathbf{4 . 0}$ & $|\boldsymbol{R e}|$ & $|\boldsymbol{R e}|$ & $\alpha=\mathbf{4 . 0}$ & $\alpha=\mathbf{3 . 9}$ \\
\hline 0.0 & 0.000000 & 0.000000 & 0.000000 & 0.000000 & 0.0000000 & 0.0000000 \\
0.1 & 0.149500 & 0.149500 & $1.44001^{-12}$ & $5.90750^{-13}$ & 0.149500 & 0.149500 \\
0.2 & 0.296009 & 0.296008 & $1.84792^{-10}$ & $2.65690^{-10}$ & 0.296008 & 0.296011 \\
0.3 & 0.436567 & 0.436561 & $2.92946^{-9}$ & $1.53937^{-8}$ & 0.436561 & 0.4365811 \\
0.4 & 0.568275 & 0.568257 & $1.53937^{-8}$ & $2.47570^{-7}$ & 0.568257 & 0.568333
\end{tabular}




$\begin{array}{lllllll}0.5 & 0.688322 & 0.688284 & 5.26460^{-9} & 2.14804^{-6} & 0.688284 & 0.688494 \\ 0.6 & 0.794012 & 0.793953 & 6.02680^{-7} & 1.28364^{-5} & 0.793953 & 0.794431 \\ 0.7 & 0.882788 & 0.882727 & 4.94654^{-6} & 5.96207^{-5} & 0.882727 & 0.883678 \\ 0.8 & 0.952261 & 0.952252 & 2.64012^{-5} & 2.30619^{-4} & 0.952252 & 0.953969 \\ 0.9 & 1.000230 & 1.000390 & 1.10933^{-4} & 7.75726^{-4} & 1.000390 & 1.003260 \\ 1.0 & 1.024720 & 1.025250 & 3.94994^{-4} & 2.33556^{-3} & 1.025250 & 1.029780\end{array}$

Table 3: Solution for different values of $\eta$ and $\alpha$ at $R=0.6$, and the corresponding absolute residual errors at $\alpha=4.0$ in case of no-slip boundary by the two methods.

\begin{tabular}{ccccccc}
\hline \multirow{2}{*}{$\eta$} & \multicolumn{3}{c}{ VIM } & \multicolumn{3}{c}{ ADM } \\
\cline { 2 - 7 } & $\alpha=\mathbf{3 . 9}$ & $\alpha=\mathbf{4 . 0}$ & $|\boldsymbol{R} \boldsymbol{e}|$ & $\boldsymbol{R} \boldsymbol{e} \mid$ & $\alpha=\mathbf{4 . 0}$ & $\alpha=\mathbf{3 . 9}$ \\
\hline 0.0 & 0.000000 & 0.000000 & 0.000000 & 0.000000 & 0.0000000 & 0.0000000 \\
0.1 & 0.149500 & 0.149500 & $7.29050^{-12}$ & $2.99094^{-12}$ & 0.149500 & 0.149501 \\
0.2 & 0.296014 & 0.296012 & $9.35462^{-10}$ & $1.34411^{-9}$ & 0.296012 & 0.296017 \\
0.3 & 0.436601 & 0.436591 & $1.48328^{-8}$ & $7.78368^{-8}$ & 0.436591 & 0.436622 \\
0.4 & 0.568412 & 0.568384 & $7.81776^{-8}$ & $1.25071^{-6}$ & 0.568384 & 0.568498 \\
0.5 & 0.688731 & 0.688673 & $1.91467^{-8}$ & $1.08378^{-5}$ & 0.688673 & 0.0688987 \\
0.6 & 0.795008 & 0.794921 & $2.95360^{-6}$ & $6.6451^{-5}$ & 0.794921 & 0.795632 \\
0.7 & 0.884903 & 0.884815 & $2.42049^{-4}$ & $2.99604^{-4}$ & 0.884815 & 0.886556 \\
0.8 & 0.956317 & 0.956311 & $1.28279^{-4}$ & $1.15562^{-3}$ & 0.956311 & 0.958846 \\
0.9 & 1.007430 & 1.007680 & $5.33793^{-4}$ & $3.87382^{-3}$ & 1.007680 & 1.011900 \\
1.0 & 1.036710 & 1.033753 & $1.87837^{-3}$ & $9.16160^{-3}$ & 1.033753 & 1.044130 \\
\hline
\end{tabular}

Table 4: Solution for different values of $\eta$ and $\alpha$ at $R=0.2$, and the corresponding absolute residual errors at $\alpha=4.0, \gamma=1.0$ in case of slip boundary by the two methods.

\begin{tabular}{ccccccc}
\hline \multirow{2}{*}{$\eta$} & \multicolumn{5}{c}{ VIM } & \multicolumn{3}{c}{ ADM } \\
\cline { 2 - 7 } & $\alpha=\mathbf{3 . 9}$ & $\alpha=\mathbf{4 . 0}$ & $|\boldsymbol{R e}|$ & $|\boldsymbol{R e}|$ & $\alpha=\mathbf{4 . 0}$ & $\alpha=\mathbf{3 . 9}$ \\
\hline 0.0 & 0.000000 & 0.000000 & 0.000000 & 0.000000 & 0.0000000 & 0.0000000 \\
0.1 & 0.075250 & 0.075250 & $1.19397^{-11}$ & $1.15647^{-11}$ & 0.075250 & 0.075250 \\
0.2 & 0.151997 & 0.151997 & $1.46091^{-9}$ & $1.42272^{-9}$ & 0.151997 & 0.151996 \\
0.3 & 0.231728 & 0.231730 & $2.41523^{-8}$ & $2.26996^{-8}$ & 0.231730 & 0.231724 \\
0.4 & 0.315911 & 0.315917 & $1.72712^{-7}$ & $1.53650^{-7}$ & 0.315917 & 0.318593 \\
0.5 & 0.405986 & 0.405998 & $7.75157^{-6}$ & $6.35861^{-7}$ & 0.405998 & 0.405930 \\
0.6 & 0.503356 & 0.503375 & $2.57633^{-6}$ & $1.87467^{-6}$ & 0.503375 & 0.503222 \\
0.7 & 0.609384 & 0.609403 & $6.92273^{-6}$ & $4.19340^{-6}$ & 0.609403 & 0.609101 \\
0.8 & 0.725384 & 0.725385 & $1.58395^{-5}$ & $7.06699^{-6}$ & 0.725385 & 0.724843 \\
0.9 & 0.852613 & 0.852560 & $3.18898^{-} 5$ & $7.55604^{-6}$ & 0.852560 & 0.851657 \\
1.0 & 0.992270 & 0.992098 & $5.77312^{-5}$ & $2.26285^{-6}$ & 0.992098 & 0.990682 \\
\hline
\end{tabular}

Table 5: Solution for different values of $\eta$ and $\alpha$ at $R=0.4$, and the corresponding absolute residual errors at $\alpha=4.0, \gamma=1.0$ in case of slip boundary by the two methods.

\begin{tabular}{ccccccc}
\hline \multirow{2}{*}{$\eta$} & \multicolumn{3}{c}{ VIM } & \multicolumn{3}{c}{ ADM } \\
\cline { 2 - 7 } & $\alpha=\mathbf{3 . 9}$ & $\alpha=\mathbf{4 . 0}$ & $|\boldsymbol{R e}|$ & $|\boldsymbol{R e}|$ & $\alpha=\mathbf{4 . 0}$ & $\alpha=\mathbf{3 . 9}$ \\
\hline 0.0 & 0.000000 & 0.000000 & 0.000000 & 0.000000 & 0.0000000 & 0.0000000 \\
0.1 & 0.075250 & 0.075250 & $1.86236^{-10}$ & $1.85035^{-10}$ & 0.075250 & 0.075250 \\
0.2 & 0.151994 & 0.151995 & $2.33752^{-8}$ & $2.27643^{-8}$ & 0.151995 & 0.151993 \\
0.3 & 0.231707 & 0.231711 & $3.86500^{-7}$ & $3.63261^{-7}$ & 0.231711 & 0.231698 \\
0.4 & 0.315823 & 0.315835 & $2.76485^{-6}$ & $2.45995^{-6}$ & 0.315835 & 0.315786
\end{tabular}




\begin{tabular}{llcllll}
0.5 & 0.405724 & 0.4057 & $1.24188^{-5}$ & $1.01911^{-5}$ & 0.405748 & 0.405614 \\
0.6 & 0.502721 & 0.502757 & $4.13358^{-5}$ & $3.01175^{-5}$ & 0.502757 & 0.502457 \\
0.7 & 0.608044 & 0.608079 & $1.11349^{-4}$ & $6.77268^{-5}$ & 0.608079 & 0.607488 \\
0.8 & 0.722830 & 0.722827 & $2.55786^{-4}$ & $1.15643^{-4}$ & 0.722827 & 0.721775 \\
0.9 & 0.848112 & 0.847998 & $5.18105^{-4}$ & $1.29590^{-4}$ & 0.847998 & 0.846262 \\
1.0 & 0.984815 & 0.984459 & $9.46358^{-5}$ & $1.08810^{-5}$ & 0.984459 & 0.981766 \\
\hline
\end{tabular}

Table 6: Solution for different values of $\eta$ and $\alpha$ at $R=0.6$, and the corresponding absolute residual errors at $\alpha=4.0, \gamma=1.0$ in case of slip boundary by the two methods.

\begin{tabular}{ccccccc}
\hline \multirow{2}{*}{$\eta$} & \multicolumn{3}{c}{ VIM } & \multicolumn{3}{c}{ ADM } \\
\cline { 2 - 7 } & $\alpha=\mathbf{3 . 9}$ & $\alpha=\mathbf{4 . 0}$ & $|\boldsymbol{R} \boldsymbol{e}|$ & $|\boldsymbol{R e}|$ & $\alpha=\mathbf{4 . 0}$ & $\alpha=\mathbf{3 . 9}$ \\
\hline 0.0 & 0.000000 & 0.000000 & 0.000000 & 0.000000 & 0.0000000 & 0.0000000 \\
0.1 & 0.075250 & 0.075250 & $9.42822^{-10}$ & $9.36742^{-12}$ & 0.075250 & 0.075250 \\
0.2 & 0.151989 & 0.151992 & $1.18341^{-7}$ & $1.15248^{-7}$ & 0.151992 & 0.151989 \\
0.3 & 0.231671 & 0.231691 & $1.95698^{-6}$ & $1.8335^{-6}$ & 0.231691 & 0.231671 \\
0.4 & 0.315680 & 0.315753 & $1.40044^{-5}$ & $1.24613^{-5}$ & 0.315753 & 0.315680 \\
0.5 & 0.405302 & 0.405500 & $6.29520^{-5}$ & $5.16793^{-5}$ & 0.405500 & 0.405302 \\
0.6 & 0.501705 & 0.502147 & $2.09836^{-4}$ & $1.53085^{-4}$ & 0.502147 & 0.501705 \\
0.7 & 0.605913 & 0.606778 & $5.66621^{-4}$ & $3.46026^{-4}$ & 0.606778 & 0.605913 \\
0.8 & 0.718799 & 0.720326 & $1.30662^{-3}$ & $5.98237^{-4}$ & 0.720326 & 0.718799 \\
0.9 & 0.841068 & 0.843560 & $2.66190^{-3}$ & $6.99132^{-4}$ & 0.843560 & 0.841067 \\
1.0 & 0.973256 & 0.977074 & $4.90297^{-3}$ & $6.98894^{-5}$ & 0.977074 & 0.973251 \\
\hline
\end{tabular}

Table 7: Absolute residual errors $|\operatorname{Re}|$ for different values of $\eta$ at $R=0.1, \alpha=4.0$ in case of no-slip and slip boundary with $\gamma=1.0$, by the NIM, PM, VIM and ADM.

\begin{tabular}{ccccccc}
\hline \multirow{2}{*}{$\eta$} & \multicolumn{3}{c}{ No-Slip $\boldsymbol{B}$} & \multicolumn{3}{c}{ Slip $\boldsymbol{B}$} \\
\cline { 2 - 7 } & NIM\&PM & VIM & ADM & NIM\&PM & VIM & ADM \\
\hline 0.0 & 0.000000 & 0.000000 & 0.000000 & 0.000000 & 0.0000000 & 0.0000000 \\
0.1 & $5.63438^{-15}$ & $5.63438^{-15}$ & $2.31759^{-15}$ & $7.27474^{-13}$ & $7.27474^{-13}$ & $7.22783^{-13}$ \\
0.2 & $7.21923^{-13}$ & $7.21923^{-13}$ & $1.03892^{-12}$ & $9.13051^{-11}$ & $9.13051^{-11}$ & $8.89284^{-11}$ \\
0.3 & $1.14405^{-11}$ & $1.14405^{-11}$ & $6.02401^{-11}$ & $1.50939^{-9}$ & $1.50939^{-9}$ & $1.41859^{-9}$ \\
0.4 & $5.97444^{-11}$ & $5.97444^{-11}$ & $9.70104^{-10}$ & $1.07916^{-8}$ & $1.07916^{-8}$ & $9.60012^{-9}$ \\
0.5 & $2.94232^{-11}$ & $2.94232^{-11}$ & $8.43342^{-9}$ & $4.84153^{-8}$ & $4.84153^{-8}$ & $3.97074^{-8}$ \\
0.6 & $2.46997^{-9}$ & $2.46997^{-9}$ & $5.05292^{-8}$ & $1.60795^{-7}$ & $1.60795^{-7}$ & $1.16926^{-7}$ \\
0.7 & $2.03243^{-8}$ & $2.03243^{-8}$ & $2.35489^{-7}$ & $4.31515^{-7}$ & $4.31515^{-7}$ & $2.60840^{-7}$ \\
0.8 & $1.09632^{-7}$ & $1.09632^{-7}$ & $9.14757^{-7}$ & $9.85311^{-7}$ & $9.85311^{-7}$ & $4.36600^{-7}$ \\
0.9 & $4.37357^{-7}$ & $4.67357^{-7}$ & $3.09276^{-6}$ & $1.97750^{-6}$ & $1.97750^{-6}$ & $4.55002^{-7}$ \\
1.0 & $1.69343^{-6}$ & $1.69343^{-6}$ & $9.36847^{-6}$ & $3.56318^{-6}$ & $3.56318^{-6}$ & $1.19842^{-7}$ \\
\hline
\end{tabular}

Table 8: Absolute residual errors $|\operatorname{Re}|$ for different values of $\eta$ at $R=0.3, \alpha=4.0$ in case of no-slip and slip boundary with $\gamma=1.0$, by the NIM, PM, VIM and ADM.

\begin{tabular}{ccccccc}
\hline & \multicolumn{3}{c}{ No-Slip $\boldsymbol{B}$} & & Slip $\boldsymbol{B}$ \\
\cline { 2 - 7 } & NIM\&PM & VIM & ADM & NIM\&PM & VIM & ADM \\
\hline 0.0 & 0.000000 & 0.000000 & 0.000000 & 0.000000 & 0.0000000 & 0.0000000 \\
0.1 & $4.55608^{-13}$ & $4.55608^{-13}$ & $1.86906^{-13}$ & $5.89262^{-11}$ & $5.89262^{-11}$ & $5.85462^{-11}$ \\
0.2 & $5.84710^{-11}$ & $5.84710^{-11}$ & $8.40956^{-11}$ & $7.39595^{-9}$ & $7.39595^{-9}$ & $7.20264^{-9}$ \\
0.3 & $9.26826^{-10}$ & $9.26826^{-10}$ & $4.87359^{-9}$ & $1.22281^{-7}$ & $1.22281^{-7}$ & $1.14927^{-7}$ \\
0.4 & $4.85819^{-9}$ & $4.85819^{-9}$ & $7.84145^{-8}$ & $8.74585^{-7}$ & $8.74585^{-7}$ & $7.78099^{-7}$ \\
0.5 & $1.90307^{-9}$ & $1.90307^{-9}$ & $6.80804^{-7}$ & $3.92681^{-6}$ & $3.92681^{-6}$ & $3.22179^{-6}$
\end{tabular}




\begin{tabular}{lllllll}
0.6 & $1.93785^{-7}$ & $1.93785^{-7}$ & $4.07194^{-6}$ & $1.30608^{-5}$ & $1.30608^{-5}$ & $9.51000^{-6}$ \\
0.7 & $1.59180^{-6}$ & $1.59180^{-6}$ & $1.89342^{-5}$ & $3.51392^{-5}$ & $3.51392^{-5}$ & $2.13295^{-5}$ \\
0.8 & $8.52601^{-6}$ & $8.52601^{-6}$ & $7.33429^{-5}$ & $8.05617^{-5}$ & $8.05617^{-5}$ & $3.61851^{-5}$ \\
0.9 & $3.59984^{-5}$ & $3.59984^{-5}$ & $2.47124^{-4}$ & $1.62693^{-4}$ & $1.62693^{-4}$ & $3.96350^{-5}$ \\
1.0 & $1.28930^{-4}$ & $1.28930^{-4}$ & $7.45555^{-4}$ & $2.95870^{-4}$ & $2.95870^{-4}$ & $7.42371^{-6}$ \\
\hline
\end{tabular}

Table 9: Absolute residual errors $|\operatorname{Re}|$ for different values of $\eta$ at $R=0.5, \alpha=4.0$ in case of no-slip and slip boundary with $\gamma=1.0$, by the NIM, PM, VIM and ADM.

\begin{tabular}{ccccccc}
\hline \multirow{2}{*}{$\eta$} & \multicolumn{3}{c}{ No-Slip $\boldsymbol{B}$} & \multicolumn{3}{c}{ Slip $\boldsymbol{B}$} \\
\cline { 2 - 7 } & NIM\&PM & VIM & ADM & NIM\&PM & VIM & ADM \\
\hline 0.0 & 0.000000 & 0.000000 & 0.000000 & 0.000000 & 0.0000000 & 0.0000000 \\
0.1 & $3.51580^{-12}$ & $3.51580^{-12}$ & $1.44218^{-12}$ & $4.54678^{-10}$ & $4.54678^{-10}$ & $4.15746^{-10}$ \\
0.2 & $4.51141^{-10}$ & $4.51141^{-10}$ & $6.48459^{-10}$ & $5.70694^{-8}$ & $5.70694^{-8}$ & $5.55778^{-8}$ \\
0.3 & $7.15258^{-9}$ & $7.15258^{-9}$ & $3.75596^{-8}$ & $9.43682^{-7}$ & $9.43682^{-7}$ & $8.86948^{-7}$ \\
0.4 & $3.76300^{-8}$ & $3.76300^{-8}$ & $6.03788^{-7}$ & $6.75191^{-6}$ & $6.75191^{-6}$ & $6.00761^{-6}$ \\
0.5 & $1.10362^{-8}$ & $1.10362^{-8}$ & $5.23539^{-6}$ & $3.03391^{-6}$ & $3.03391^{-6}$ & $2.49016^{-5}$ \\
0.6 & $1.44776^{-6}$ & $1.44776^{-6}$ & $3.12585^{-5}$ & $1.01056^{-4}$ & $1.01056^{-4}$ & $7.36777^{-5}$ \\
0.7 & $1.18733^{-5}$ & $1.18733^{-5}$ & $1.45021^{-4}$ & $2.72557^{-4}$ & $2.72557^{-4}$ & $1.66113^{-4}$ \\
0.8 & $6.31480^{-5}$ & $6.31480^{-5}$ & $5.60162^{-4}$ & $6.27313^{-4}$ & $6.27313^{-4}$ & $2.85430^{-4}$ \\
0.9 & $2.64053^{-4}$ & $2.64053^{-4}$ & $1.88097^{-3}$ & $1.27436^{-3}$ & $1.27436^{-3}$ & $3.26825^{-4}$ \\
1.0 & $9.34686^{-4}$ & $9.34686^{-4}$ & $5.65177^{-3}$ & $2.33762^{-3}$ & $2.33762^{-3}$ & $3.76189^{-6}$ \\
\hline
\end{tabular}

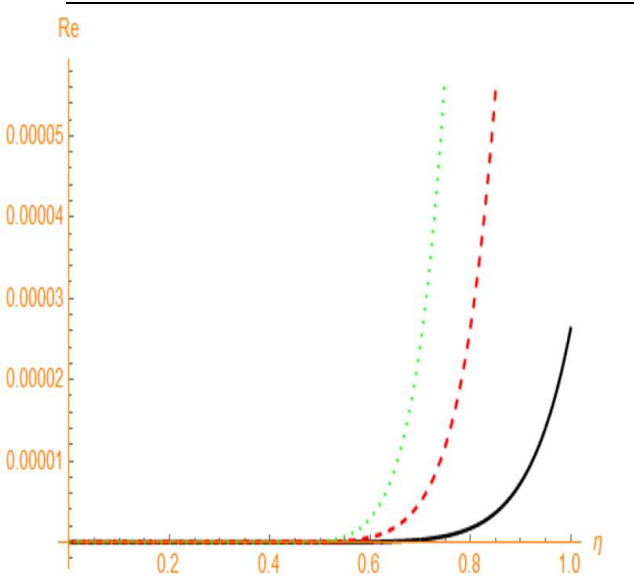

Figure 2(a): Residuals for $R=0.2$ (continuous curve), 0.4 (dashed curve), 0.6 (dotted curve) at $\alpha=4.0$, by VIM in case of no-slip boundary.

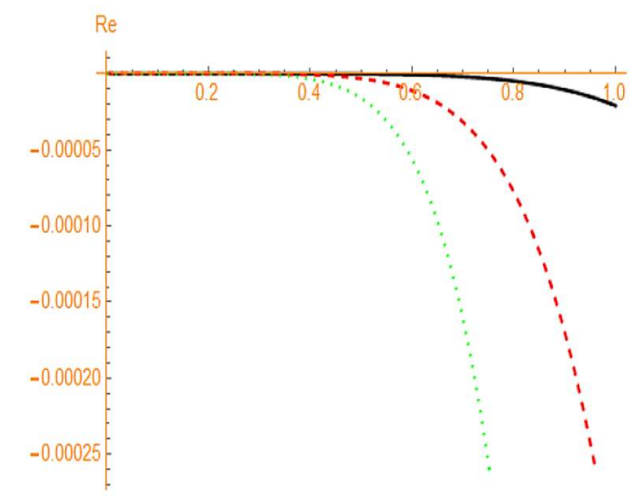

Fig. 2(b): Residuals for $R=0.2$ (continuous curve), 0.4 (dashed curve), 0.6 (dotted curve) at $\alpha=4.0, \gamma=0.9$, by ADM in case of slip boundary.

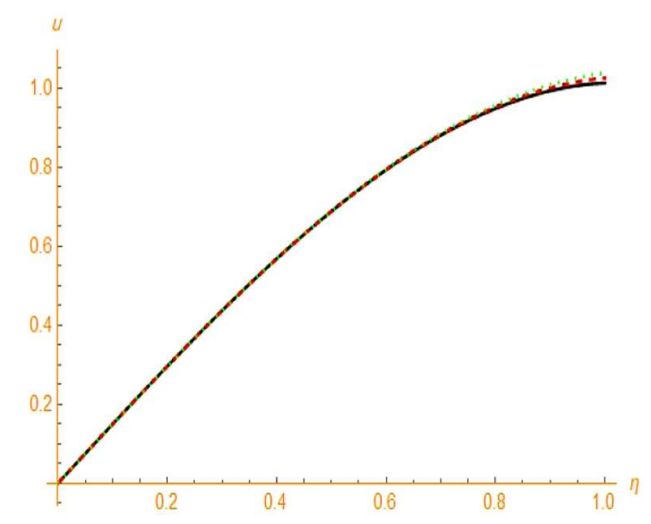

Fig. 3(a): Velocity profiles (Normal velocity) for $R=0.2$ (continuous curve), 0.4 (dashed curve), 0.6 (dotted curve) at $\alpha=4.0$, by VIM in case of no-slip boundary. 


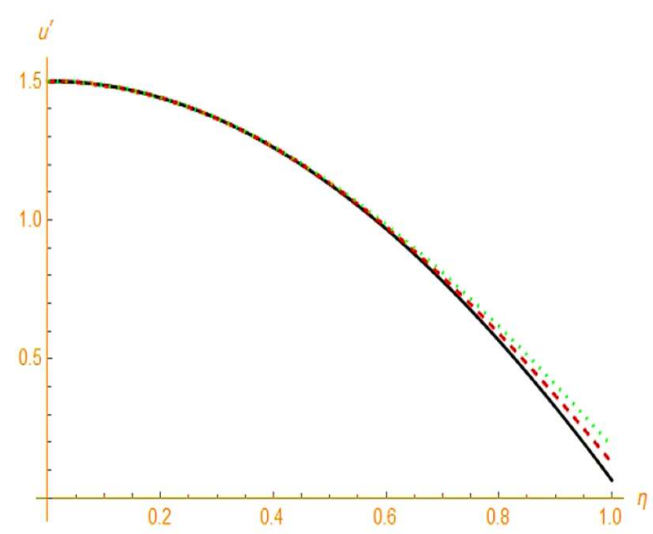

Fig. 3(b): Velocity profiles (Longitudinal Normal velocity) for $R=0.2$ (continuous curve), 0.4 (dashed curve), 0.6 (dotted curve) at $\alpha=4.0$, by VIM in case of no-slip boundary.

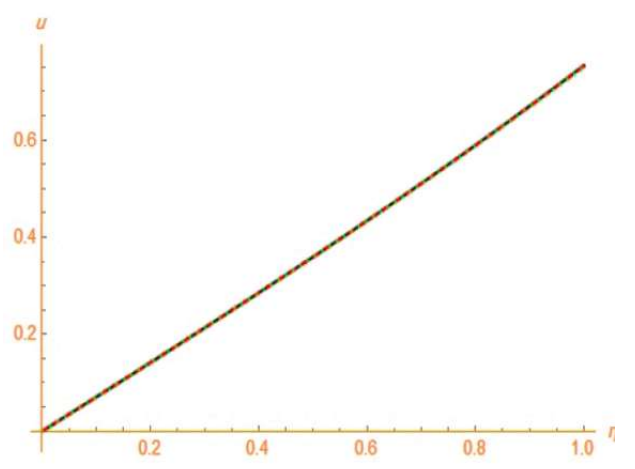

Fig. 4(a): Velocity profiles (Normal velocity) for $R=0.2$ (continuous curve), 0.4 (dashed curve), 0.6 (dotted curve) at $\alpha=4.0, \gamma=0.9$, by ADM in case of slip boundary.

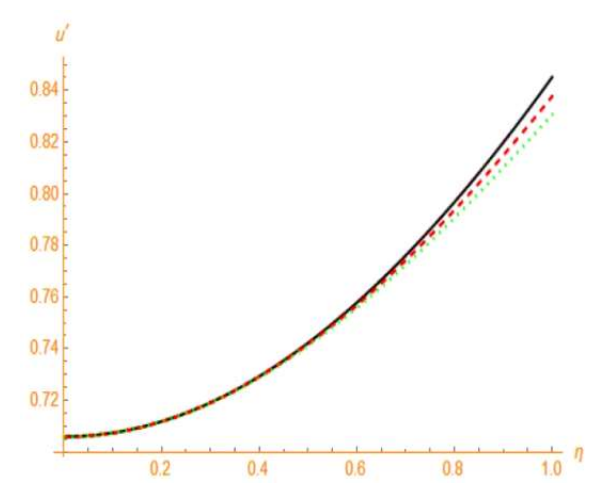

Fig. 4(b): Velocity profiles (Longitudinal velocity) for $R=0.2$ (continuous curve), 0.4 (dashed curve), 0.6 (dotted curve) at $\alpha=4.0, \gamma=0.9$, by ADM in case of slip boundary.

\section{Conclusion}

In this chapter, an analytical solution for an unsteady axisymmetric flow of in compressible, nonconducting Newtonian fluid squeezed between two circular plates in fractional form is obtained using of the VIM and ADM in cases of no-slip and slip boundaries. Analysis of the residual errors confirms that the VIM and ADM are almost identical and efficient schemes. Convergence of the considered methods is confirmed by absolute residual errors for different values of the Reynolds number $R$. The comparisons for the $|\mathrm{Re}|$ for VIM, ADM, and NIM \& PM confirmed that the results of the four methods are identical. Therefore, we concluded that the considered methods can be effectively used in various fields of science and engineering as thy give better results in terms of accuracy.

\section{Conflict of Interest}

The authors declare that there are no conflicts of interest regarding the publication of this paper.

\section{References}

[1] M. J. Stefan, Versuch Über die scheinbare Adhäsion, Sitzungsber., Abt II, Öster. Akad. Wiss., Math.-Naturwiss. Kl., 69 (1874) 713721.

[2] J. F. Thorpe, in W. A. Shaw, Developments in Theoretical and Applied Mechanics, Pergamon Press, Oxford, 1976.

[3] P. S. Gupta and A. S. Gupta, Squeezing flow between parallel plates, wear, 45 (1997), 177185.

[4] X. J. Ran, Q. Y. Zhu and Y. Li, An explicit series solution of the squeezing flow between two infinite plates by means of the homotopy analysis method, Comm. Nonlinear Sci. Numer. Simul., 14 (2009), 119-132.

[5] M. M. Rashidi, A. M. Siddiqui and M. Asadi, Application of homotopy analysis method to the unsteady squeezing flow of a secondgrade fluid between circular plates, Math. Prob. Engin., vol. 2010, Article ID 706840, 18 pages, (2010).

[6] D. Yao, V. L. Virupaksha and B. Kim, Study on squeezing flow during nonisothermal embossing of polymer microstructures, Polymer Engin. Sci., 45 (5) (2005), 625-660.

[7] P. J. Leider and R. B. Bird, Squeezing flow between parallel disks, I. Theoretical Analysis, Ind. Eng. Chem. Fundam., 13 (1974), 336-341.

[8] R. L. Verma, A numerical solution for squeezing flow between parallel channels, Wear, 72 (1981), 89-95. 
[9] P. Singh, V. Radhakrishnan and K. A. Narayan, Squeezing flow between parallel plates, Ingenieu-archives, 60 (1990), 274281.

[10] A. A. Hemeda and E. E. Eladdad, Iterative methods for solving the fractional form of unsteady axisymmetric squeezing fluid flow with slip and no-slip boundaries. Advances of Mathematical Physics, Volume 2016, Article ID 6021462, 11 pages, DOI: $10.115 / 2016 / 6021462$.

[11] M. Qayyum, H. Khan, M. T. Rahim and I. Ullah, Analysis of unsteady axisymmetric squeezing fluid flow with slip and no-slip boundaries using OHAM, Math, Prob. Eng., 2015 (2015), 11 pages.

[12] M. Qayyum, H. Khan, M. T. Rahim and I. Ullah, Modeling and analysis of unsteady axisymmetric squeezing fluid flow through porous medium channel with slip boundary, PLOS ONE, 10 (3) (2015).

[13] M. Sheikoleslami, D. D. Ganji and H. R. Ashorynejad, Investigation of squeezing unsteady nanofluid flow using ADM, Powder Technology, 239 (2013), 256-265.

[14] M. Sheikholeslami and D. D. Ganji, Heat transfer of $\mathrm{Cu}$-water nanofluid flow between parallel plates, Powder Technology, 235 (2013), 8873-879.

[15] I. Ullah, H. Khan and M. T. Rahim, Approximation of first grade NHD squeezing fluid with slip boundary condition using DTM and OHAM, Math. Prob. Eng., vol. 2013, Article ID 816262, 9 pages, 2013.

[16] R. J. Grimm, Squeezing flows of Newtonian liquid films an analysis include the fluid inertia, Appl. Sci. Research, 32 (2) (1976), 149-166.

[17] H. M. Laun, M. Rady and O. Hassager, Analytical solutions for squeeze flow with partial wall slip, J. Non-Newtonian Fluid Mech., 81 (1999), 1-15.

[18] M. M. Rashidi, A. M. Siddiqui and M. T. Rastegari, Analytical solution of squeezing flow between two circular plates, Int. J. Comput. Methods Eng. Sci. Mech., 13 (5) (2012), 342-249.

[19] M. K. Yazdi and P. H. Tehrani, The emergy balance to nonlinear oscillations via Jacobi collocation method, Alexandria Engin. J., 54 (2015), 99-103.

[20] H. Askari, E. Esmailzadeh and A. Bararu, A unfied approach for nonlinear vibration analysis of curved structures using nonuniform rational B-spline representation,
Journal of Sound and Vibration, 353 (2015), 292-307.

[21] D. Younesian, H. Askari, Z. Saadatnia and M. Kalam Yazdi, Frequency analysis of strongly nonlinear generalized Duffing oscillators using He's frequency-amplitude formulation and He's energy balance method, Computer and Mathematics with Applications, 59 (9) (2010), 3222-3228.

[22] A. Mirzabeigy, M. K. Yazdi and M. H. Naschi, Approximate periodic solution for the nonlinear Helmholtz-Duffing oscillator via analytical approaches, Int. J. Comp. Math., vol. 2014, Article ID 939623, 3 pages, (2014).

[23] M. K. Yazdi, H. Ahmadian, A. Mirzabeigy and A. Yildirim, Dynamic analysis of vibrating systems with nonlinearities, Commun, Theor. Phys., 57 (2012), 183-187.

[24] A. A. Hemeda, Variational iteration method for solving wave equation, Comput. Math. Appl., 56 (2008), 1948-1953.

[25] A. A. Hemeda, Variational iteration method for solving nonlinear partial differential equations, Chaos Solitons \& Fractals, 39 (2009), 1297-1303.

[26] J. H. He, Variational iteration method- a kind of non-linear analytic technique: some examples, Int. J. Nonlinear Mech., 34 (1999), 699-708.

[27] G. -C. We and D. Baleanu, Variational iteration method for fractional calculus- a universal approach by Laplace transform, Advances in Difference equations, 2013, 2013:18, DOI:10.1186/1687-1847-2013-18.

[28] Z. Odibat and S. Momani, The variational iteration method: An efficient scheme for handling fractional partial differential equations in fluid mechanics, Comput. Math. Appli., 58 (2009), 2199-2208.

[29] Z. Odibat and S. Momani, Numerical methods for nonlinear partial differential equations of fractional order, Appli. Math. Model., 32 (2008), 28-39.

[30] T. Özis and A. Yildirim, Comparison between Adomian method and He's homotopy perturbation method, Comput. Math. Appli., 65 (5) (2008), 1216-1224.

[31] A. Wazwaz, A new algorithm for calculating Adomian polynomials for nonlinear operator, Appli. Math. Compute., 111 (2000), 53-69.

[32] S. -P. Yan, H. Jafari and H. K. Jassim, Local fractional Adomian decomposition and function decomposition method for Laplace equation within local fractional operators, 
Advances in Mathematical Physics, vol. 2014 (2014), Article ID 161580, 7 pages.

[33] D. Baleanu, J. A. Tenreiro, C. Cattani, M. C. Baleanu and X. -J. Yang, Local fractional variational iteration and decomposition methods for wave equation on cantor sets within local fractional operators, Abstract and Applied analysis, vol. 2014 (2014), Article ID 535048, 6 pages.

[34] R. A. Van Gorder, The variational iteration method is a special case of the homotopy analysis method, Appl. Math. Lett., 45 (2015), 81-85.

[35] I. Podlubny, Fractional Differential Equations, Academic Press, San Diego, 1999.

[36] M. Caputo, Elasticita E Dissipaxione, ZaniChelli, Bologna, Italy, 1969. 